\title{
RAVE spectroscopy of luminous blue variables in the Large Magellanic Cloud
}

\author{
U. Munari ${ }^{1}$, A. Siviero ${ }^{1,5}$, O. Bienaymé ${ }^{2}$, J. Binney ${ }^{3}$, J. Bland-Hawthorn ${ }^{4}$, R. Campbell ${ }^{5,6}$, K. C. Freeman ${ }^{7}$,
} J. P. Fulbright ${ }^{8}$, B. K. Gibson ${ }^{9}$, G. Gilmore ${ }^{10}$, E. K. Grebel ${ }^{11}$, A. Helmi ${ }^{12}$, J. F. Navarro ${ }^{13}$, Q. A. Parker ${ }^{6}$, W. Reid ${ }^{6}$, G. M. Seabroke ${ }^{10,14}$, A. Siebert ${ }^{2,5}$, M. Steinmetz ${ }^{5}$, F. G. Watson ${ }^{15}$, M. Williams ${ }^{5,7}$, R. F. G. Wyse ${ }^{8}$, and T. Zwitter ${ }^{16}$

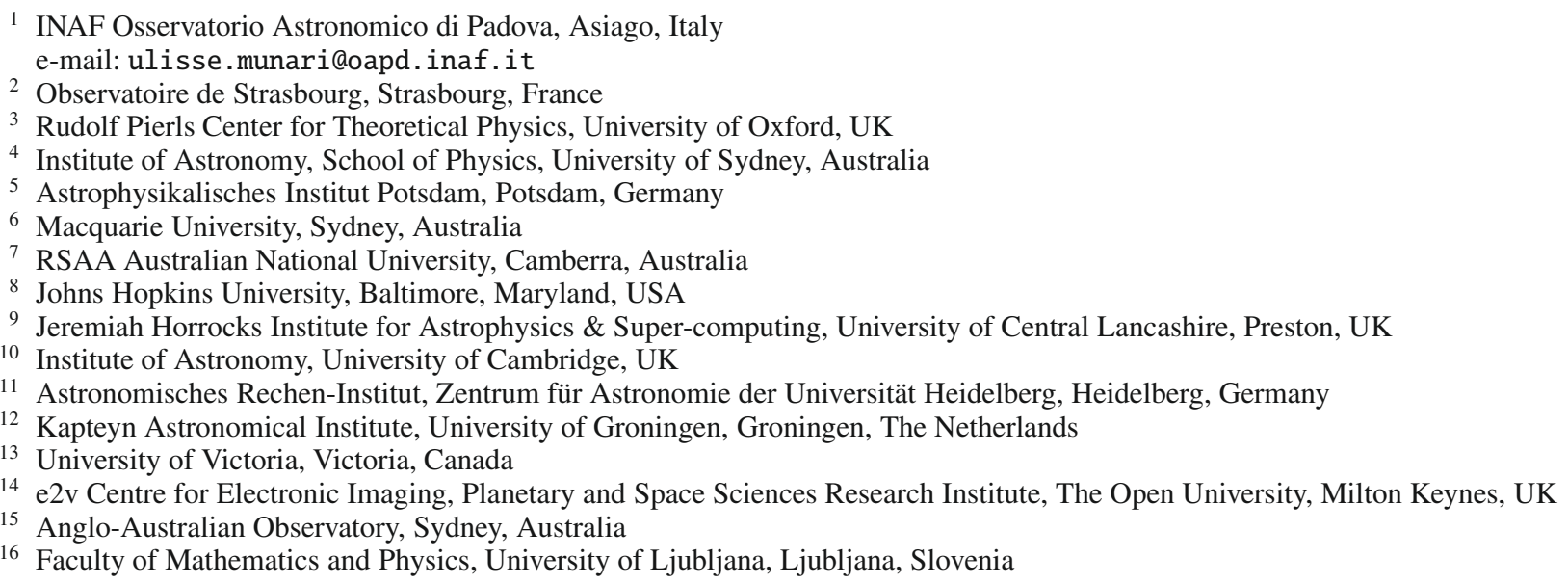

Received 28 April 2009 / Accepted 2 June 2009

ABSTRACT

\begin{abstract}
Context. The RAVE spectroscopic survey for galactic structure and evolution obtains $8400-8800 \AA$ spectra at 7500 resolving power at the UK Schmidt Telescope using the $6 \mathrm{dF}$ multi-fiber positioner. More than $3000009 \leq I_{\mathrm{C}} \leq 12$ and $|b| \geq 25^{\circ}$ southern stars have been observed to date.

Aims. This paper presents the first intrinsic examination of stellar spectra from the RAVE survey, aimed at evaluating their diagnostic potential for peculiar stars and at contributing to the general understanding of luminous blue variables (LBVs).

Methods. We used the multi-epoch spectra for all seven LBVs observed, between 2005 and 2008, in the Large Magellanic Cloud (LMC) by the RAVE survey.

Results. We demonstrate that RAVE spectra possess significant diagnostic potential when applied to peculiar stars and, in particular, LBVs. The behaviour of the radial velocities for both emission and absorption lines, and the spectral changes between outburst and quiescence states are described and found to agree with evidence gathered at more conventional wavelengths. The wind outflow signatures and their variability are investigated, with multi-components detected in S Doradus. Photoionisation modelling of the rich emission line spectrum of R 127 shows evidence of a massive detached ionised shell that was ejected during the 1982-2000 outburst. Surface inhomogeneities in the nuclear-processed material, brought to the surface by heavy mass loss, could have been observed in S Doradus, even if alternative explanations are possible. We also detect the transition from quiescence to outburst state in R 71. Finally, our spectrum of R 84 offers one of the clearest views of its cool companion.
\end{abstract}

Key words. stars: emission-line, Be - stars: winds, outflows - stars: Wolf-Rayet - galaxies: Magellanic Clouds - surveys

\section{Introduction}

RAVE (RAdial Velocity Experiment) is an ongoing digital spectroscopic survey of stars in the magnitude interval $9 \leq I_{\mathrm{C}} \leq 12$, distributed over the whole southern sky at galactic latitudes $|b| \geq 25^{\circ}$. Spectra are recorded over the 8400-8800 ̊ range, at a resolving power $\sim 7500$, with the UK Schmidt telescope feeding light to a spectrograph via the 6-degree Field (6dF) 150 fiber positioner. Via the determination of radial velocities, chemistries, temperatures, and gravities for a large number of high-latitude stars, the overarching science driver for the survey is the investigation of the structure and evolution of the Milky Way. At the time of writing, RAVE has observed over 300000 stars, Data Releases 1 and 2 have been published (Steinmetz et al. 2006; Zwitter et al. 2008), the third is coming (Siebert et al. 2008), and scientific exploitation of the data has begun (e.g. Smith et al. 2007; Siebert et al. 2008; Seabroke et al. 2008; Munari et al. 2008; Veltz et al. 2008).

The essentially unbiased selection of targets guarantees that in addition to normal stars, peculiar ones are also observed. The type of peculiarities most easy to recognise with RAVE spectra are the presence of emission lines, absorption line splitting in double-line binaries, signatures of stellar winds, and combination spectra. The present paper is the first to explore the 
Table 1. List of the LBVs observed by RAVE in the LMC, their date of observation and optical brightness at that time, and the radial velocities of the associated emission and absorption lines.

\begin{tabular}{cccccc}
\hline \hline HD & name & obs. date & $V$ & $\begin{array}{c}\text { RV em. } \\
\left(\mathrm{km} \mathrm{s}^{-1}\right)\end{array}$ & $\begin{array}{c}\text { RV abs. } \\
\left(\mathrm{km} \mathrm{s}^{-1}\right)\end{array}$ \\
\hline 35343 & S Dor & 20050320 & 9.55 & $293.0 \pm 1.4$ & $299.0 \pm 0.2$ \\
& & 20060323 & 9.45 & $297.4 \pm 1.0$ & $266.5 \pm 2.7$ \\
269006 & R 71 & 20060323 & 10.70 & $199 \pm 11$ & $186.7 \pm 2.1$ \\
& & 20060927 & 10.65 & $193.4 \pm 2.4$ & $205.4 \pm 2.2$ \\
269445 & R 99 & 20060323 & 11.50 & $289.6 \pm 5.1$ & \\
& & 20060927 & 11.55 & $288.4 \pm 3.4$ & \\
269929 & R 143 & 20060927 & 11.15 & & $286.6 \pm 2.4$ \\
269216 & S 88 & 20050320 & 10.40 & & $249.1 \pm 2.6$ \\
269227 & R 84 & 20060927 & 11.80 & & $254.8 \pm 3.4$ \\
269858 & R 127 & 20060927 & 10.40 & $276.5 \pm 0.4$ & \\
\hline
\end{tabular}

performance of RAVE in relation to the physics of peculiar stars, in particular, the Luminous Blue Variables (LBVs) of the Large Magellanic Cloud (LMC). As part of its tiling strategy, RAVE observed the region of sky occupied by the LMC in March 2005, March 2006, September 2006, and February 2008. As LBVs are intrinsically very luminous, and those belonging to the LMC are brighter than the $I_{C} \leq 12$ survey limit (indeed, many possess an HD identifier), they naturally enter the RAVE target set. A list of the LBVs observed by RAVE in the LMC is given in Table 1.

LBVs were first identified in M31 and M33 by Hubble \& Sandage (1953) as extremely luminous hot stars that underwent irregular photometric variability of modest amplitude (a few mag) over timescales of years. Their bolometric magnitude generally exceeds -9.5 , corresponding to a luminosity of the order $\sim 10^{6} L_{\odot}$, close to their Eddington Limit. During quiescence, the LBVs obey a tight temperature-luminosity relation, calibrated as $\log L / L_{\odot}=1.37 \log T_{\text {eff }}-0.03$ by van Genderen (2001). The existence of such a relation, their extreme intrinsic brightness and easy identification (the latter provided by their variability), make LBVs important players in the cosmic distance ladder game (e.g. Davidson et al. 1989). The LBVs are sometimes also called Hubble-Sandage variables or S Dor variables from the archetype of the class, and are believed to be the immediate progenitor of Wolf-Rayet stars of the classic N-type (nitrogen rich and hydrogen poor). The LBV phase is not particularly longlasting ( $\sim 0.025 \mathrm{Myr})$ and, together with the paucity of massive stars, accounts for the relative rarity of LBVs (Crowther 2007). The number of recognised LBVs differs from author-to-author, ranging from 20 to 50 throughout the entire Local Group (e.g. van Genderen 2001; Weis 2003). The most famous LBVs include $\eta$ Car and P Cyg, in the Galaxy, and S Dor in the LMC.

After the main sequence phase, the most massive stars do not evolve all the way to the reddest part of the HR diagram but instead enter a phase of very high mass loss $\left(10^{-4}-10^{-5} M_{\odot} \mathrm{yr}^{-1}\right)$ and reverse their evolution back toward hotter effective temperatures, becoming LBVs. The position of this turning point depends upon the star's luminosity and sets what is called the Humphreys-Davidson Limit (cf. Humphreys \& Davidson 1994). The LBVs lay to the hotter side of this $T_{\text {eff }}$ limit, which may be crossed during eruptive events of the most massive objects.
The evolutionary sequence leading to the formation of LBVs depends on the mass of the progenitor. For stars more massive than $75 M_{\odot}$ it is $\mathrm{O} \rightarrow \mathrm{WN}(\mathrm{H}$-rich $) \rightarrow \mathrm{LBV} \rightarrow \mathrm{WN}(\mathrm{H}$-poor $) \rightarrow \mathrm{WC}$ $\rightarrow$ SNIc, while for those of initial mass $40 \leq M \leq 75 M_{\odot}$ it is $\mathrm{O} \rightarrow \mathrm{LBV} \rightarrow \mathrm{WN}(\mathrm{H}-$ poor) $\rightarrow \mathrm{WC} \rightarrow$ SNIc (cf. Crowther 2007, and references therein). Direct spectroscopic evidence for LBV progenitors transitioning to supernovae is being obtained (eg. SN 2005gj - Trundle et al. 2008). Only lower-mass O-type stars can experience a red supergiant phase just before or after the LBV phase (Szeifert et al. 1996; Smith et al. 2004), their evolutionary sequence being $\mathrm{O} \rightarrow \mathrm{LBV} / \mathrm{RSG} \rightarrow$ $\mathrm{WN}(\mathrm{H}$-poor) $\rightarrow$ SNIb (where RSG $=$ Red Supergiant). This is supported by the simultaneous presence of LBVs and RSGs observed in Westerlund 1 , the most massive young open cluster of our Galaxy, with $1 \times 10^{5} M_{\odot}$ total mass and $\leq 25 M_{\odot}$ turn-off mass (Clark et al. 2005). To the best of our knowledge, this simultaneity is not known to occur elsewhere.

The large amount of mass lost during the LBV phase is the critical stage that a very massive star must pass through before becoming a WR star. Mass ejection via major outbursts is far more efficient than that via steady winds, as most notably undergone by $\eta$ Car during the 19th century when the star shed several $M_{\odot}$ in less than a decade (Smith \& Owocki 2006). The mass lost by the central LBV star frequently gives rise to circumstellar nebulae (e.g. Langer et al. 1999; Weis 2003) that turn out to be enriched in processed material, such as nitrogen and helium, coming from the stellar interior.

During the quiescent phase, the optical spectra of LBVs are characterised by effective temperatures ranging from 12000 to $30000 \mathrm{~K}$ (B-spectral types) and strong emission lines of hydrogen, HeI, FeII, CaII and other singly ionised metals, often with P-Cygni profiles when observed at sufficiently high resolution (cf. Kenyon \& Gallagher 1985). The mass loss rate in quiescence is smaller and the wind faster. While not well-understood an LBV occasionally passes through an "outburst" phase, during which the luminosity remains the same as in quiescence, while in tandem the mass loss rate increases and the wind velocity decreases. This pushes outward the pseudo-photosphere, with a consequent increase in the effective radius and decrease in the surface temperature (whose Wien peak moves from the ultraviolet toward the optical region). At outburst maximum, the effective temperature is $\sim 7500-8500 \mathrm{~K}$ regardless of the LBV luminosity and temperature in quiescence, and the spectrum turns to that of A-type supergiants, while [FeII], HeI emission lines, and P-Cygni absorptions weaken or disappear. Wolf (1989; see Vink 2008, for an update) pointed out the existence of an amplitude-luminosity relation for LBV eruptions and called $S$ Doradus instability strip the region of the HR diagram occupied by LBVs in quiescence. Stothers \& Chin (1995) suggested the existence of a period-luminosity relation of the form $M_{\mathrm{bol}}=-12.5( \pm 0.5)+2.4( \pm 0.5) \log P$ where $P$, expressed in yrs, is the typical interval from one eruption to the next.

\section{RAVE Spectra of the LBVs}

The spectra that RAVE collected for the LBVs in the LMC are presented in Figs. 1-3, where the ordinate scale is kept constant for an easier inter-comparison. Differences in $\mathrm{S} / \mathrm{N}$ among the multi-epoch spectra of the same star are mainly due to differences in the throughputs of different fibers and in the sky conditions. The radial velocities derived from the absorption and emission lines are given in Table 1 (for comparison, the heliocentric barycentric velocity of the LMC is $+278 \mathrm{~km} \mathrm{~s}^{-1}$, Mateo 1998 , 


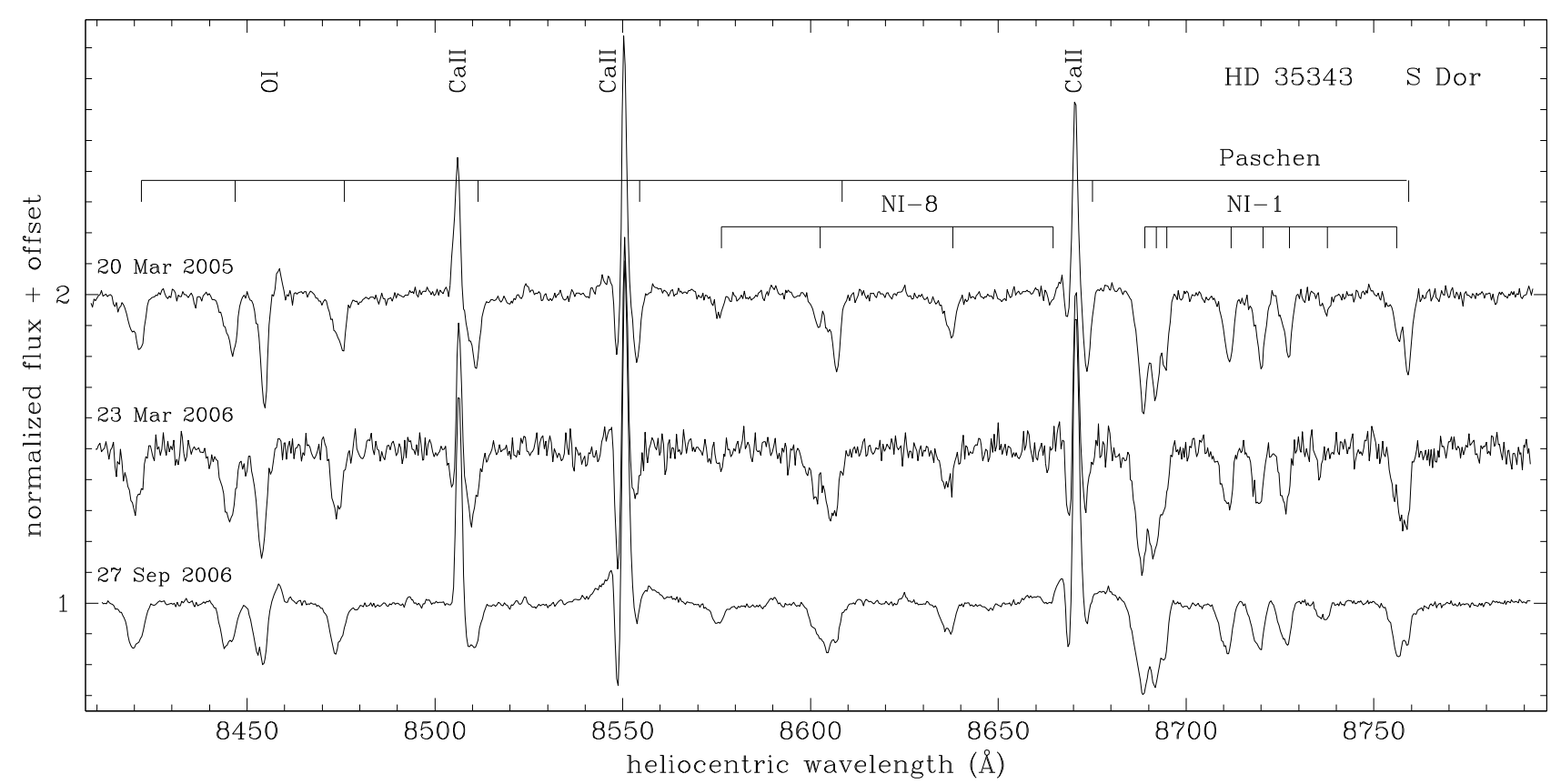

Fig. 1. Multi-epoch RAVE spectra of S Doradus. Paschen and NI (multiplets 1 and 8) absorption lines are indicated by comb markings.

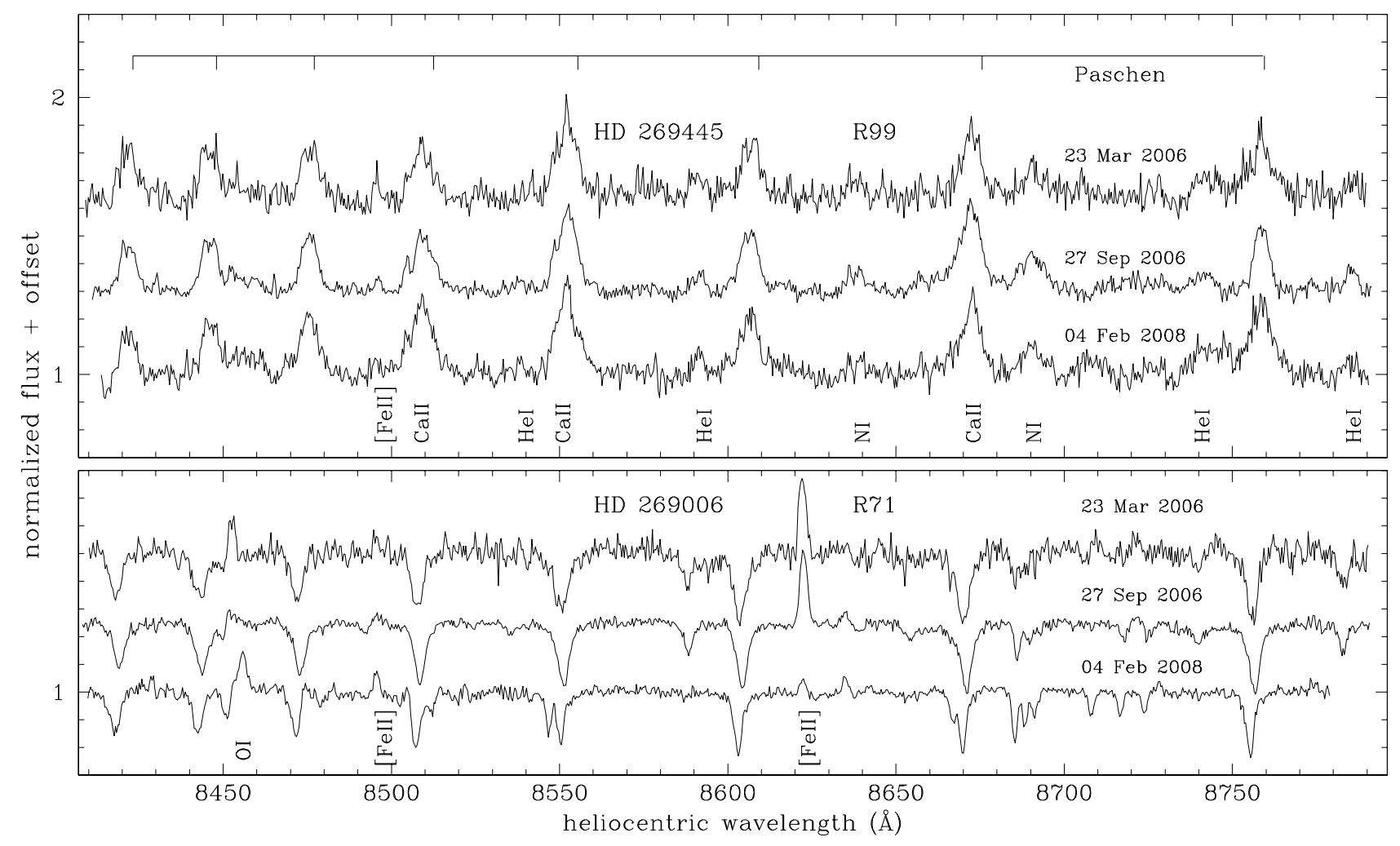

Fig. 2. Multi-epoch RAVE spectra of R 99 and R 71.

although significantly variable across the face of the galaxy). Table 1 also lists the $V$-magnitude at the time of the RAVE observation as estimated by consulting various databases of variable star amateur observers (AAVSO, VSNET, VSOLJ) and the ASAS Survey database (Pojmanski 2002).

These observations indicate that the RAVE spectra are well suited to detect and study peculiar stars, and LBVs in particular, confirming early investigations in this wavelength interval by
Munari (1999, 2002, 2003). The peculiarities stands out well in terms of absorption features, wind outflow signatures, and emission lines, and will now be considered in turn.

\subsection{Emission lines}

The ionised circumstellar gas is easily revealed by the rich emission line spectrum observable over the RAVE wavelength 


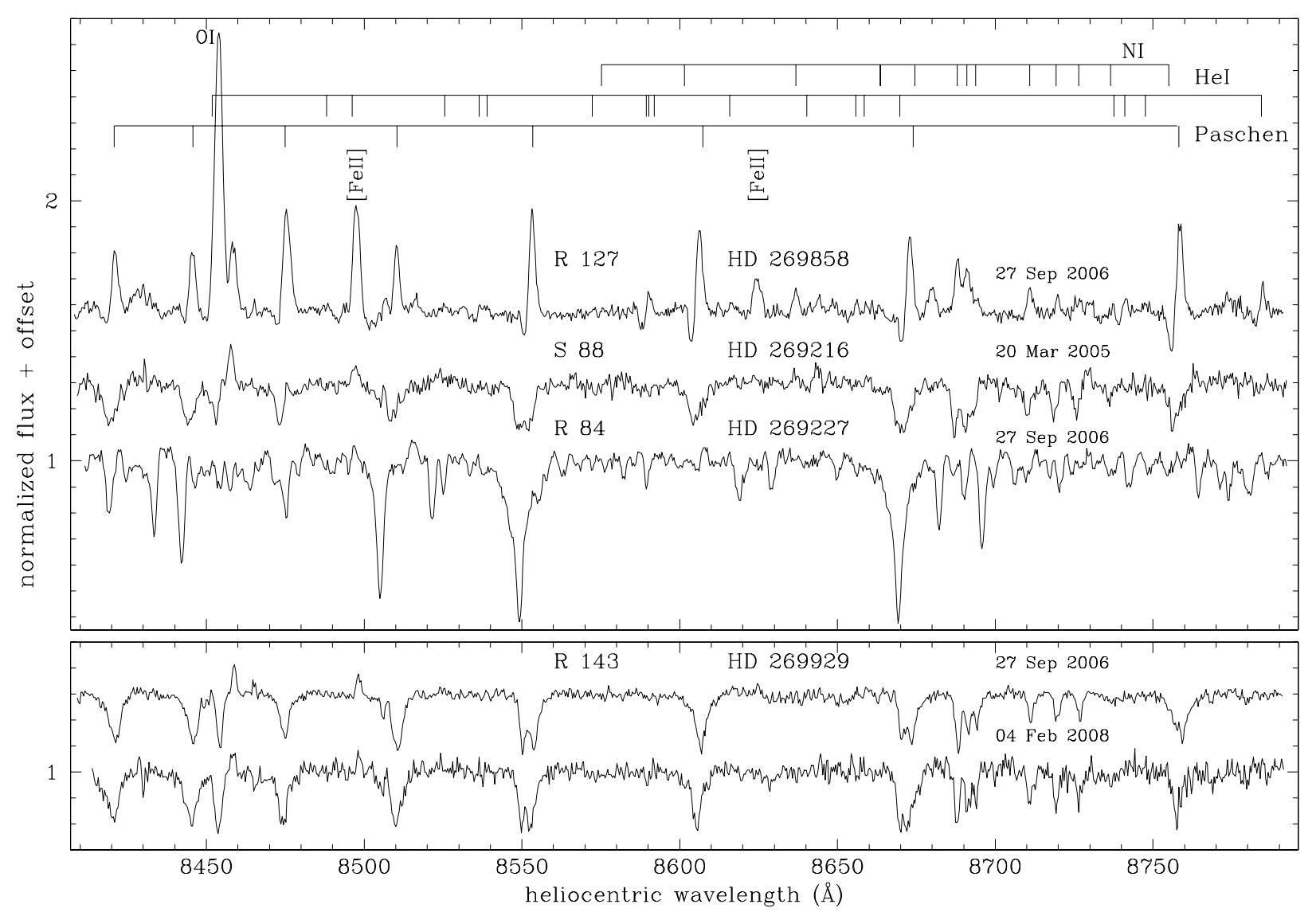

Fig. 3. RAVE spectra of R 127, S 88, R 84, and R 143.

regime. The principal ions contributing emission lines at these wavelengths are HI, HeI, CaII, NI, CII, CIII, OI, SI, [FeII], FeII, FeIII, [CIII], and [CIIII]. The emission lines identified and measured in the LBV RAVE spectra of Figs. 1-3 are listed in Table 2. They all belong either to OI, NI, [FeII], HeI, CaII or to the Paschen series of hydrogen.

The emission lines in RAVE spectra of LBVs are fairly "sharp". The expansion velocity derived from the width of the profiles (corrected for the FWHM(PSF) $=40 \mathrm{~km} \mathrm{~s}^{-1}$ instrumental resolution) is on average $25 \mathrm{~km} \mathrm{~s}^{-1}$, which is the same as obtained from observations at more classical optical wavelengths (Weis 2003). The only exception is R 99 (cf. Fig. 2), whose expansion velocity from Paschen lines in RAVE spectra is $\sim 90 \mathrm{~km} \mathrm{~s}^{-1}$, comparable to the Balmer line-based results of Nota et al. (1996).

The intensity of the OI 8446 emission line can be boosted by fluorescence pumping from absorption of hydrogen Lyman$\beta$ photons by OI in its ground state, as first pointed out by Bowen (1947). For the Lyman- $\beta$ fluorescence to be effective, the optical depth in $\mathrm{H} \alpha$ should be large, owing to the population of the $n=2$ level by trapped Lyman- $\alpha$ photons. Such a fluorescence pumping is at work in R 127 (cf. Fig. 3), but not in R 99 (cf. Fig. 2), as derived by comparing the intensity of OI 8446 emission with that of the Paschen series of hydrogen, following Strittmatter et al. (1977) analytical modelling of the pumping mechanism.

\subsection{Wind outflows}

Significant mass loss from a central star is revealed in our LBV spectra by pronounced P-Cygni profiles. A selection of such
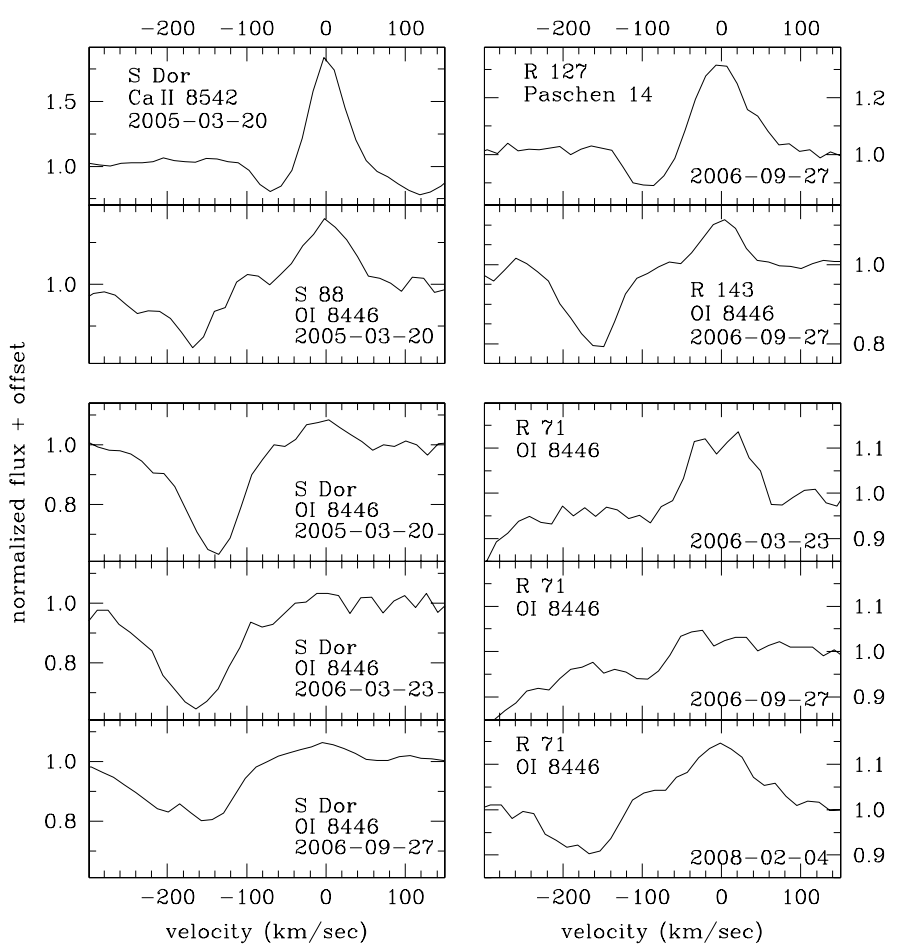

Fig. 4. Sample of P-Cygni profiles of LBVs from RAVE spectra.

profiles is presented in Fig. 4. The line showing a wind-driven P-Cygni profile most consistently is that of OI $8446 \AA$. 
Table 2. Equivalent width of the emission lines identified and measured in the RAVE spectra of LBVs in the LMC.

\begin{tabular}{|c|c|c|c|c|}
\hline \multirow[t]{2}{*}{$\lambda_{\mathrm{o}}(\AA)$} & \multirow[t]{2}{*}{ Ion } & \multicolumn{3}{|c|}{ Equivalent width $(\AA)$} \\
\hline & & $\begin{array}{c}\text { R 99 } \\
(27-09-06)\end{array}$ & $\begin{array}{c}\text { R 127 } \\
(27-09-06)\end{array}$ & $\begin{array}{c}\text { S Dor } \\
(20-03-05)\end{array}$ \\
\hline 8413 & P-19 & 0.974 & 0.556 & \\
\hline 8438 & P-18 & 1.167 & 0.606 & \\
\hline 8446 & {$[\mathrm{OI}]$} & 0.216 & 3.445 & 0.148 \\
\hline 8451 & $?$ & & 0.825 & \\
\hline 8467 & P-17 & 1.270 & 1.057 & \\
\hline 8490 & {$[\mathrm{FeII}]$} & 0.156 & 1.023 & \\
\hline 8498 & CaII & \multirow{3}{*}{1.613} & & 0.734 \\
\hline 8502 & $\mathrm{P}-16$ & & 0.460 & \\
\hline 8518 & $\mathrm{HeI}$ & & 0.080 & \\
\hline 8531 & $\mathrm{HeI}$ & 0.098 & 0.107 & \\
\hline 8542 & CaII & \multirow[b]{2}{*}{2.065} & & 1.138 \\
\hline 8545 & P-15 & & 0.875 & \\
\hline 8583 & $\mathrm{HeI}$ & 0.269 & 0.143 & \\
\hline 8598 & P-14 & 1.640 & 0.725 & \\
\hline 8615 & $\mathrm{HeI}$ & & 0.326 & \\
\hline 8617 & {$[\mathrm{FeII}]$} & \multirow{3}{*}{0.573} & 0.068 & \\
\hline 8629 & NI & & 0.188 & \\
\hline 8636 & $?$ & & 0.101 & \\
\hline 8662 & CaII & \multirow{3}{*}{2.851} & & 0.968 \\
\hline 8665 & P-13 & & 0.642 & \\
\hline 8671 & $\mathrm{HeI}$ & & 0.290 & \\
\hline 8680 & NI & \multirow{7}{*}{1.377} & 0.392 & \\
\hline 8683 & $\mathrm{NI}$ & & 0.471 & \\
\hline 8686 & $\mathrm{NI}$ & & 0.132 & \\
\hline 8703 & NI & & 0.240 & \\
\hline 8712 & NI & & 0.126 & \\
\hline 8719 & $\mathrm{NI}$ & & 0.090 & \\
\hline 8729 & NI & & 0.063 & \\
\hline 8734 & $\mathrm{HeI}$ & 0.394 & 0.122 & \\
\hline 8750 & $\mathrm{P}-12$ & 1.232 & 0.845 & \\
\hline \multirow[t]{2}{*}{8777} & $\mathrm{HeI}$ & 0.300 & 0.177 & \\
\hline & & $\begin{array}{c}\text { R } 71 \\
(23-03-06)\end{array}$ & $\begin{array}{c}\text { S 88 } \\
(20-03-05)\end{array}$ & $\begin{array}{c}\text { R } 143 \\
(27-09-06)\end{array}$ \\
\hline 8446 & [OI] & 0.369 & 0.242 & 0.162 \\
\hline 8490 & [FeII $]$ & & 0.160 & 0.142 \\
\hline 8617 & {$[\mathrm{FeII}]$} & 0.743 & & 0.053 \\
\hline
\end{tabular}

In a P-Cygni profile, the emission component traces the systemic velocity (e.g. Castor \& Lamers 1979), so that the blueward displacement of the absorption component relates to the velocity of the wind at the position in the expanding medium where the given line forms (Lamers \& Cassinelli 1999). The RAVE spectra in Fig. 4 show that the wind velocity from OI lines is slow and quite similar in each of the surveyed LBVs, ranging from 130 to $210 \mathrm{~km} \mathrm{~s}^{-1}$. These velocities closely match those derived from high resolution optical and ultraviolet spectra (e.g. Leitherer et al. 1985; Crowther \& Willis 1994; GarciaLario et al. 1998). The RAVE spectra demonstrate that the velocity, intensity, and shape of the wind absorption component is highly variable for any given object, as illustrated in Fig. 4 by the OI profiles of S Dor and R 71 at three distinct epochs. For $\mathrm{R} 71$, the wind absorption emerged only in the outburst spectrum of 4 February 2008, contrary to the normal behaviour of LBVs. S Dor showed significant variability of the OI absorption component during a period of constant brightness. The $\mathrm{S}$ Dor spectrum of 27 September 2006 shows two distinct wind components, at 140 and $210 \mathrm{~km} \mathrm{~s}^{-1}$. Wind absorption profiles characterised by multi-components (variable with time) have been previously observed in high-resolution optical spectra of R 127 (Stahl et al. 1983) and AG Car (Stahl et al. 2001), extending over the same range of velocities detected in the RAVE spectra of S Dor.

The high variability of the absorption components of P-Cygni profiles reflects the complexity of wind condition and structure in massive and LBV stars. The winds of LBVs are both aspherical and inhomogeneous, as revealed by the highly variable polarisation measured by Davies et al. (2005). The amount of mass loss (in $M_{\odot} \mathrm{yr}^{-1}$ ) in such stars relates to both luminosity (in $L_{\odot}$ ) and effective temperatures $(\mathrm{K})$ as $\log \dot{M}=$ $1.738 \log L-1.352 \log T_{\text {eff }}-9.547$, following the calibration of Lamers \& Leitherer (1993). The wind velocity field is usually assumed to follow a $\beta$-type velocity law $v(r)=v_{\infty}\left(1-R_{\star} / r\right)^{\beta}$ (cf. Schmutz et al. 1991). The smaller the $\beta$, the shorter the distance the wind needs to travel before reaching the terminal velocity. Guo \& $\mathrm{Li}$ (2007) found that $0.5 \leq \beta \leq 0.7$ fits the observations of LBVs in quiescence, while $\beta \geq 1.5$ is required for bright states. Thus, in quiescent LBVs, $v_{\infty}$ is reached within a very few stellar radii, beyond which the gas density declines as $\rho(r)=\rho\left(r_{\circ}\right)\left[r_{\circ} / r\right]^{2}$, while during bright states $v_{\infty}$ is reached only at a much greater distance.

A cursory inspection of the spectra of S Dor in Fig. 1 either (i) could suggest the presence of weak and variable emission cores within Paschen absorption lines; or (ii) could indicate that $\mathrm{CaII}$ emission lines appear in emission within the adjacent Paschen 13, 15, and 16 lines, with no associated true P-Cygni absorption component. That this is not the case is illustrated by Fig. 5, where a "zoomed" view is provided for one of the S Dor spectra in Fig. 1. Figure 5 clearly illustrates how the the presence of weak emission cores within Paschen absorption lines is actually due to the interplay with nearby NI absorption lines, and how CaII lines do really present a P-Cygni absorption component not associated with the wings of adjacent Paschen lines.

\subsection{Absorption spectrum and chemical abundances}

The classification of the absorption spectrum of B-A-F stars at the RAVE wavelengths is straightforward, as illustrated by the observed MK spectral atlases of Munari \& Tomasella (1999), Cenarro et al. (2001), le Borgne et al. (2003), Marrese et al. (2003), and Andrillat et al. (1995), as well as the LTE synthetic spectral atlas by Castelli \& Munari (2001) and the atlas of nonLTE synthetic spectra of OB super-giants by Clark et al. (2005). The absorption spectra of LBVs can be classified using these atlases, with the caveat that both markedly non-solar surface abundance patterns and the pseudo-photosphere being placed in a moving, partially ionised wind, can confuse the picture (see Jaschek \& Jaschek 1987, for a self-consistent definition of classification criteria attributes). Nevertheless, the distinction between quiescent (B-type absorption spectra) and outburst states (A-type absorption spectra), as well as the signatures of type Ia luminosity class are straightforward to recognise.

Significant mass loss removes the external layers of massive stars, exposing progressively deeper internal regions and reveals the mixing with nuclearly processed material, which manifests 


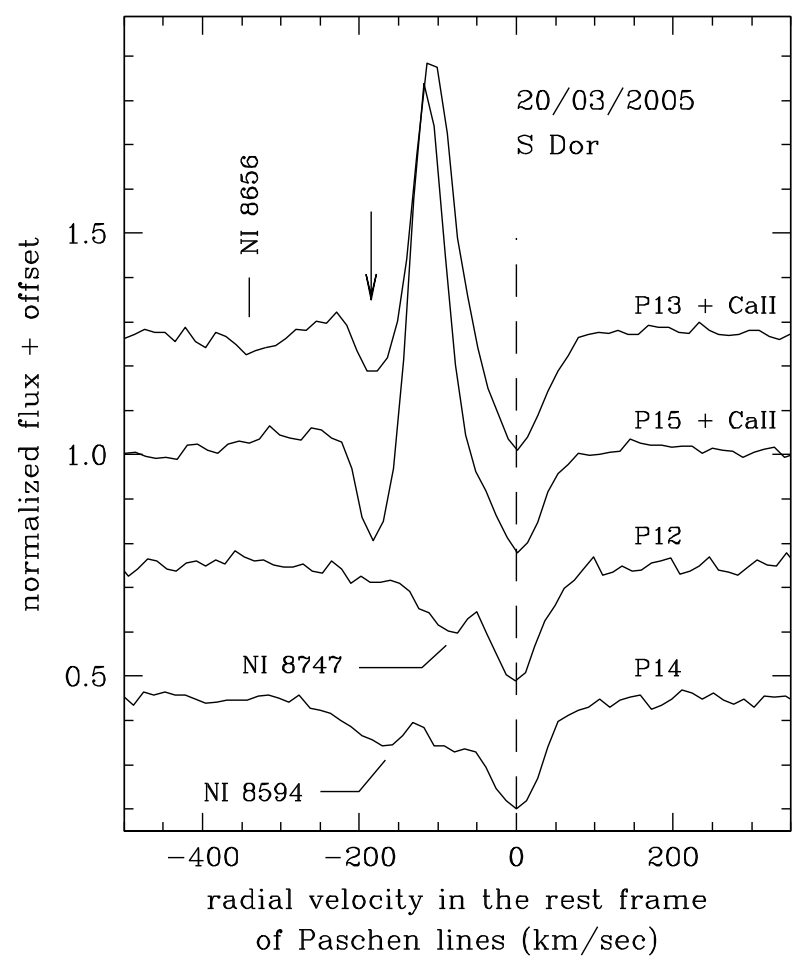

Fig. 5. Expanded view of the $S$ Doradus spectrum of Fig. 1 near the Paschen 12 + NI 8747, Paschen 13 + CaII 8662, and Paschen $15+$ CaII 8542 features. The arrow points to the absorption component of the CaII P-Cygni profiles.

as a depletion of hydrogen, oxygen, and carbon, and an enhancement of helium and (especially) nitrogen (cf. Meynet \& Maeder 2005). Hydrogen, helium, nitrogen, and oxygen possess strong lines within the RAVE wavelength interval, at the effective temperatures and gravities characterizing LBVs. Actually, multiplets 1 and 8 over the RAVE range are the strongest NI features observable over the whole optical range (cf. Jaschek $\&$ Jaschek 1995). The hydrogen deficiency and nitrogen enrichment is quite obvious in the RAVE spectra of LBVs presented in this paper. There is no temperature, luminosity, and metallicity combination, for which a normal stellar atmosphere with a solar abundance pattern can display NI lines stronger than Paschen ones as, for example, seen in the S Dor spectra of Fig. 1. This is both true for LTE and non-LTE synthetic spectra (Castelli \& Munari 2001; Clark et al. 2005); for this to occur, it would require, at the same time, both an enhancement in nitrogen and depletion in hydrogen.

\subsection{Radial velocities}

Table 2 presents the radial velocities of the LBVs measured from the RAVE spectra. The accuracy of the wavelength scale of RAVE spectra is $\sim 1.0 \mathrm{~km} \mathrm{~s}^{-1}$ (Zwitter et al. 2008). The radial velocities of emission lines for the objects with multi-epoch observations are stable within a dispersion of $2.3 \mathrm{~km} \mathrm{~s}^{-1}$ for S Dor, $2.4 \mathrm{~km} \mathrm{~s}^{-1}$ for $\mathrm{R} 71$, and $0.5 \mathrm{~km} \mathrm{~s}^{-1}$ for $\mathrm{R} 99$. This is consistent with the long-term stability seen by Thackeray (1974) in LBV emission line radial velocities (within a few $\mathrm{km} \mathrm{s}^{-1}$ ), regardless of the phase (quiescent or outburst). Note that the observations of R 71 in Table 2 cover both the quiescent and outburst phases.

The radial velocity of the emission lines in Table 2 are comparable to those derived from high-resolution optical spectra at various epochs. Wasselink (1956) derived $+295 \mathrm{~km} \mathrm{~s}^{-1}$ for $\mathrm{S}$ Dor, Thackeray (1974) found $+195 \mathrm{~km} \mathrm{~s}^{-1}$ for $\mathrm{R} 71$, and Weis reported $+286 \mathrm{~km} \mathrm{~s}^{-1}$ for $\mathrm{R} 99$. The situation for $\mathrm{R} 127$ is less clear with a low accuracy value of $+284 \mathrm{~km} \mathrm{~s}^{-1}$ claimed by Feast et al. (1960), $+292 \pm 9 \mathrm{~km} \mathrm{~s}^{-1}$ by Stahl et al. $(1983),+267 \mathrm{~km} \mathrm{~s}^{-1}$ by Weis (2003), and $+274 \pm 1 \mathrm{~km} \mathrm{~s}^{-1}$ by Stahl \& Wolf (1986). The RAVE value of $+276.5 \pm 0.4 \mathrm{~km} \mathrm{~s}^{-1}$ lies within $2.5 \mathrm{~km} \mathrm{~s}^{-1}$ of both the mean of these four determinations $\left(+279 \pm 5 \mathrm{~km} \mathrm{~s}^{-1}\right)$ and the most accurate value amongst them $(+274 \pm 1)$, again consistent with a long-term constant value. Small differences in velocity of the emission component of a P Cygni profile are expected when the line strength is variable.

On the contrary, the radial velocities of the absorption lines given in Table 2 are highly variable from epoch-to-epoch, by several tens of $\mathrm{km} \mathrm{s}^{-1}$. This relates to the variable positioning of the pseudo-photosphere within the accelerating portion of the out-flowing wind, driven at least in part by instability and inhomogeneities in the mass loss rate (Davies et al. 2005). This "breathing" pseudo-photosphere is also responsible for the low amplitude, irregular photometric variability (with timescales of months to a few years) which is persistent in LBVs both in quiescence and outburst phases. The light-curves presented by Sharov (1975), Stahl et al. (1984), Humphreys \& Davidson (1994), and Lamers (1995), amongst others, provide clear examples of this restless variability. This variability can be further appreciated by comparing the RAVE values in Table 2 with the velocities given by Feast et al. (1960) for S Dor $\left(+213 \mathrm{~km} \mathrm{~s}^{-1}\right), \mathrm{R} 71(+198)$, R 143 (+263) and R 84 (+262).

\subsection{Photoionisation analysis}

The RAVE spectrum of R 127 provides a sufficient number of emission lines (cf. Table 2) to attempt modelling of its photoionisation structure. To this aim, we have used the CLOUDY code (Ferland et al. 1998) to evaluate the prevailing physical conditions in R 127 at the time of the RAVE observations, and to assess the diagnostic potential of emission lines observable in the RAVE wavelength regime. Even though this exercise did provide consistent results (as outlined below), some degree of caution must be exercised, as the number of emission lines observable over the restricted wavelength range of RAVE is somewhat limited, and only a fraction of them is currently treated by CLOUDY (most notably, the NI and HeI lines are ignored). This type of modelling is best carried out by combining emission lines from the entire optical, ultraviolet, and infrared regimes, which provides access to a larger set of emission lines from more ions and from a wider range of excitation conditions. Such an expanded set of input data could also allow exploration of more complex, multi-component emission environments, than the simple onecomponent geometry adopted here.

RAVE optical fibers have an aperture of 7 arcsec, which corresponds to $1.7 \mathrm{pc}$ at distance of the LMC. This is large enough to include a contribution (by unknown relative proportion) to the observed spectrum from the central star, its wind, the extended circumstellar nebula, any parent H II region, and any close optical companion (many LBVs are members of massive and dense young stellar clusters). Concerning R 127 , it is surrounded by a faint nebula 5 arcsec in diameter. Judging from spatially-resolved long slit spectra presented by Weis (2003), the light from this nebula (clearly visible on HST images obtained through an $\mathrm{H} \alpha+[\mathrm{NII}]$ narrow-band filter) contributes a negligible fraction of the light compared with the unresolved central source. Similarly, there is is no parent HII region in which 
Table 3. Comparison between computed and observed emission lines. Intensities are scaled to Paschen 14.

\begin{tabular}{cccc}
\hline \hline$\lambda_{\mathrm{o}}(\AA)$ & Ion & \multicolumn{2}{c}{ Relative flux } \\
\cline { 3 - 4 } & & observed & computed \\
8446 & {$[\mathrm{OI}]$} & 4.75 & 4.73 \\
8498 & CaII & $\sim 0$ & $<0.01$ \\
8542 & CaII & $\sim 0$ & $<0.01$ \\
8545 & $\mathrm{P}-15$ & 1.21 & 1.61 \\
8598 & $\mathrm{P}-14$ & 1.00 & 1.00 \\
8617 & {$[$ FeII] } & 0.09 & 0.08 \\
8662 & CaII & $\sim 0$ & $<0.01$ \\
8665 & $\mathrm{P}-13$ & 0.89 & 1.14 \\
8750 & $\mathrm{P}-12$ & 1.17 & 1.29 \\
\hline
\end{tabular}

$\mathrm{R} 127$ is embedded, so this is also not likely to be a problem. The adaptive optic observations by Heydary-Malayeri et al. (2003) resolved R 127 into a dense cluster of stars, with two close optical companions to the LBV: one is 1 arcsec away and 5 mag fainter, and the other is 3 arcsec away and 2.7 mag fainter. The spectrum of the latter star (as presented by Heydary-Malayeri et al. 2003) is deficient in emission lines, and consequently, is not contaminating the LBV emission line spectrum. In conclusion, only the ionised mass outflow from the LBV is contributing to the emission lines observed in the RAVE spectra.

Walborn et al. (1982) have reviewed the recent photometric and spectroscopic history of $\mathrm{R} 127$, and found it entered a long-lasting outburst phase in the early-1980s, peaking in the $V$-band at 8.9 mag during 1988-1991, and then slowly declining toward quiescence (by the time of the RAVE observations in 2006, R 127 had reached this quiescent state). According to the analysis of Guo \& Li (2007), the wind of LBVs in quiescence is accelerated to its terminal velocity within a few stellar radii. Therefore, the vast majority of its mass is located external to the acceleration zone and is expanding at constant velocity. In our modelling, we thus adopted $\rho(r)=\rho\left(r_{\circ}\right)\left[r_{\circ} / r\right]^{2}$ for the dependence of density on distance from the central star. To be conservative, we also explored other density dependencies (e.g. wind accelerated to terminal velocity over significant distances, density independent of distance from the central star), but abandoned them ultimately due to the resulting inferior results. We assumed Case B nebular conditions (Osterbrock \& Ferland 2006), spherical geometry with the photoionising star at the center, and chemical homogeneity throughout the gas. The stellar photosphere was assumed to emit as a black body. The chemical partition was taken to be solar for all elements except those allowed to vary. The initial value for the overall metallicity was taken to be $[\mathrm{M} / \mathrm{H}]=-0.5$. Having no observed emission line from carbon, and CLOUDY not treating the $\mathrm{He}$ and $\mathrm{N}$ lines recorded over the RAVE spectral range, we assumed (and kept fixed) their abundance to the average value found in other LBVs - i.e., a $10 \times$ enhancement for $\mathrm{N}$ and $2.5 \times$ for $\mathrm{He}$, and a $0.2 \times$ depletion for C (cf. Crowther \& Willis 1994, and references therein). These fixed values for $\mathrm{He}, \mathrm{N}$, and $\mathrm{C}$ are close to those found by Lamers et al. (2001) for the external, spatially resolved, nebula around R 127.

The emission lines that we used in the modelling, along with their observed and computed flux ratios, are shown in Table 3. The difference between computed and observed fluxes is $\sim 10 \%$. This can be regarded as an excellent match, reflecting the limitations/capabilities of such photoionisation modelling, regardless
Table 4. Basic parameters for the central star and ionised circumstellar material derived by photoionisation modelling of the emission line spectrum of R 127.

\begin{tabular}{ll}
\hline \hline$T_{\mathrm{BB}}^{\text {eff }}$ & $15000 \mathrm{~K}$ \\
$R_{\mathrm{BB}}$ & $140 R_{\odot}$ \\
$L_{\mathrm{BB}}$ & $9 \times 10^{5} L_{\odot}\left(M_{\mathrm{bol}}=-10.1\right)$ \\
$r_{\text {in }}^{\text {neb }}$ & $19 R_{*}(13.4 \mathrm{AU})$ \\
$r_{\text {out }}^{\text {neb }}$ & $27 R_{*}(17.6 \mathrm{AU})$ \\
$\rho_{\text {in }}^{H}$ & $1.7 \times 10^{10} \mathrm{~cm}^{-3}$ \\
$\rho_{\text {out }}^{H}$ & $8.5 \times 10^{9} \mathrm{~cm}^{-3}$ \\
$N_{\text {in }}^{e}$ & $2.1 \times 10^{10} \mathrm{~cm}^{-3}$ \\
$N_{\text {out }}^{e}$ & $8.1 \times 10^{5} \mathrm{~cm}^{-3}$ \\
$T_{\text {in }}^{e}$ & $10750 \mathrm{~K}$ \\
$T_{\text {out }}^{e}$ & $2200 \mathrm{~K}$ \\
$\xi$ & 0.92 \\
{$[\mathrm{M} / \mathrm{H}]$} & -0.4 \\
$\mathrm{H} / \mathrm{H}_{\odot}$ & 0.50 \\
$\mathrm{O} / \mathrm{O}_{\odot}$ & 0.39 \\
$\mathrm{Fe} / \mathrm{Fe} \odot$ & 1.88 \\
$\mathrm{Ca} / \mathrm{Ca}_{\odot}$ & 2.21 \\
$\mathrm{He} / \mathrm{He}_{\odot}$ & $(2.5)$ \\
$\mathrm{N} / \mathrm{N}_{\odot}$ & $(10)$ \\
$\mathrm{C} / \mathrm{C}_{\odot}$ & $(0.2)$ \\
\hline &
\end{tabular}

of the object under analysis (e.g. novae, planetary nebulae, galactic nuclei, etc.)

The output parameters of the modelling are given in Table 4, where $\xi$ is the filling factor (the ratio of filled-to-total volume in the ejecta). The derived temperature, radius, and luminosity are consistent with other modelling attempts in the literature, despite being based on completely different sets of input data. For example, Stahl et al. (1983) derived from optical and IUE high resolution observations of $\mathrm{R} 127$ (during the quiescence phase of the early-1980s), $T_{\text {eff }}=16000 \mathrm{~K}, R=150 R_{\odot}$, and $M_{\mathrm{bol}}=-10.6$. The hydrogen and oxygen under-abundances we have obtained are similar to those found in other LBVs (Lennon et al. 1993; Crowther \& Willis 1994; Venn 1997; Smith 1997), in R 127 itself (Lamers et al. 2001), and expected by theoretical evolution models of massive stars that include rotation (Meynet \& Maeder 2005).

The results in Table 4 indicate that the emission lines of $\mathrm{R} 127$ originate in an ionised shell detached from the central star, extending from 19 to 27 stellar radii. The mass within this ionised shell is:

$M_{\text {shell }}=\frac{\xi}{X} \int_{r_{\text {in }}}^{r_{\text {out }}} 4 \pi r^{2} \rho^{H}(r) \mathrm{d} r=1.33 \times 10^{-3} M_{\odot}$

where $X$ is the hydrogen mass fraction, and $\rho^{H}(r)=\rho_{\text {in }}^{H}\left(r_{\text {in }} / r\right)^{2}$.

The existence and meaning of such a detached shell deserves comment, as fits squarely into the coherent picture emerging as a result of the full suite of data associated with $\mathrm{R} 127$.

Weis (2003) and Smith et al. (1998) determined from high resolution spectra that the bulk velocity of the mass outflow during the recent outburst was $\sim 10 \mathrm{~km} \mathrm{~s}^{-1}$. Material expanding with this velocity should have left R 127 during 2000, in order to have reached (by the time of our RAVE observations) a distance from the central star equal to that of the inner border of the ionised shell in our model. It is interesting to note that the lightcurve 
of Walborn et al. (1982) shows R 127 started a rapid decline in brightness in 1999 that ended in 2000 when the star reached its "stable" quiescent brightness. Thus, we argue that the end of the outburst in 2000 brought with it also the cessation of an enhanced mass loss phase, producing a cavity around the star that was only tenuously filled by the much lighter wind characterizing the subsequent quiescence conditions.

The material at the external ionisation boundary of the shell (17.6 AU), expanding at $10 \mathrm{~km} \mathrm{~s}^{-1}$, should have left R $1272.5 \mathrm{yr}$ before the material at the inner edge. The mass loss rate necessary to produce the observed shell is therefore

$\dot{M}=\frac{M_{\text {shell }}}{\Delta t}=5 \times 10^{-4} M_{\odot} / \mathrm{yr}$

This is a magnitude larger than the mass loss rate derived by Stahl et al. (1983) for R 127 before the outburst. A large increase in the mass loss is a characteristic feature of LBVs during outburst phases (Humphreys \& Davidson 1994). The material lost earlier in the outburst had, by the time of the RAVE observations, moved exterior to the ionisation boundary, where the irradiation from the central star is no longer effective in ionising the gas, and therefore it was no longer contributing to the emission line spectrum.

This scenario is well matched by the spectroscopic evolution that Walborn et al. (1982) presents for R 127. The 1999-2000 timeframe, during which the star ejected the material comprising the ionised shell seen in the RAVE spectra, was characterised by the disappearance of P-Cygni absorption profiles and by the appearance of very sharp emission line profiles. These are indicative an extended atmosphere moving outward at low velocity. Later spectra, when the significant mass loss had finished and the detaching of the massive shell had initiated, started showing a progressive broadening of the emission lines and re-emergence of P-Cygni profiles characteristic of a less-significant and faster mass loss regime. The winds of LBVs are line-driven, and the drop in outward velocity and large increase in mass loss rate during outburst phases is caused by the driving effect of FeIII lines below the sonic point as the effective temperature decreases (Vink et al. 1999). The bifurcated wind regimes between quiescence and outburst phases, referred to as the "bi-stability" jump (Pauldrach \& Puls 1990), is a defining characteristic of the S Dor instability strip (Vink \& de Koter 2002).

\section{Remarks on individual objects}

\subsection{S Doradus}

The three RAVE spectra presented in Fig. 1 were obtained while $\mathrm{S}$ Dor was stable during its outburst, at $V \sim 9.5 \mathrm{mag}$. Much like the Thackeray (1965) analysis, the continuum of the RAVE spectra of S Dor can be readily classified as A2/3 $\mathrm{Ia}^{+}$e, by comparison with the Munari \& Tomasella (1999) atlas.

Quite interesting is the reversal in the ratio of equivalent width (REW) of hydrogen and NI absorption lines that occurred between 20 March 2005 and 27 September 2006 spectra (cf. Fig. 1). The ratio changed from $\mathrm{REW}=0.7$ to $\mathrm{REW}=3.0$. This happened at stable brightness, surface temperature, and wind outflow velocity, while the intensity of the CaII emission components increased by $1.6 \times$ between the two dates. It would be tempting to interpret the reversal of REW as an indication of chemical inhomogeneities in the nuclearly processed material progressively exposed at the stellar surface by the on-going significant mass loss. To support this interpretation additional and higher resolution observations are however necessary. In fact, the observed variation in the intensity of the CaII emission lines could suggest the presence of unresolved and variable emission cores as a (contributing) reason for the observed changes in the equivalent widths of nitrogen and hydrogen lines.

\section{2. $R 71$}

The LBV nature of R 71 was discovered by Thackeray (1974), who reported that the star oscillates between 9.8 and 10.9 mag (see also Lamers 1995) and that the corresponding spectral types ranged from A1eq to B2.5Iaep. During bright optical phases, Balmer lines show deep P-Cygni profiles and the [FeII] emission lines disappear.

The first two RAVE observations of $\mathrm{R} 71$ were obtained when the star was in quiescence at $V \sim 10.7$, while the third was during outburst, at $V=9.95$. The corresponding spectral changes in Fig. 2 bear a striking resemblance to the behavior described by Thackeray (1974). The RAVE quiescence spectra are clearly those of an early B super-giant (strong HeI absorption lines, no OI, and broader Paschen lines) with superimposed strong [FeII] emission lines. The outburst spectrum is that of an early A super-giant, where the HeI lines have disappeared and CaII and OI have appeared, along with the reinforcement of NI and the sharpening of Paschen lines. Similar to the optical spectra, in the outburst spectrum recorded by RAVE, the [FeII] has nearly disappeared and OI has developed a strong P-Cygni absorption component.

\section{3. $R 84$}

R 84 is an enigmatic object, suggested to be a dormant LBV by Crowther et al. (1995). Walborn (1977) classified the blue optical spectrum as OIafpe, with many strong emission lines (including HeII 4686) and P-Cygni profiles. Higher resolution blue optical spectroscopy by Stahl et al. (1985) show the same characteristics. Breysacher et al. (1999, and references therein) summarised the determinations of the physical parameters obtained by various authors, which cluster around a temperature of $30000 \mathrm{~K}$, a radius of $30 R_{\odot}$, a luminosity $6 \times 10^{5} L_{\odot}$ and a mass loss rate $3 \times 10^{-4} M_{\odot} / \mathrm{yr}$

The spectral appearance at longer wavelengths is however in conflict with that at the bluest end of the optical range. TiO absorption bands were observed shortward of $6000 \AA$ by Sanduleak \& Philip (1977) on objective prism plates, and by Cowley \& Hutchings (1978) in slit spectra. Allen \& Glass (1976) found no evidence of them in far red spectra, but reported that the MgI- $b$ triplet could be present weakly in absorption and that the IR colors implied the presence of a cool super-giant. Stahl et al. (1984) noted that the IR colour excess was too large to result only from a late-type companion, and suggested the presence of dust in $\mathrm{R} 84$, which was confirmed by the mid-IR observations of Glass (1984).

These apparently contradicting reports on the spectral appearance support a binary or optical pair nature of R 84 . Some photometric variability of one or both component stars is the probable cause of their brightness ratio to change with time. Schmutz et al. (1991) argued strongly in favor of the LBV and the cool star being merely along the same line of sight and not members of a binary system. It should be noted that adaptive optic observations by Heydari-Malayeri et al. (2003), which have a resolution limit of $0.12 \operatorname{arcsec}$ (corresponding to $6000 \mathrm{AU}$ 
at the distance of the LMC), did not resolve the central star. Stahl et al. (1984) found a good fit to the IUE-optical-IR spectral energy distribution by combining an M4Ia spectrum with a B0Ia spectrum, and with the addition of a circumstellar dust component. The spectral type of the cool super-giant was given as $\sim \mathrm{M} 2$ by Cowley \& Hutchings (1978).

The RAVE spectrum of R 84 in Fig. 3 presents the clearest view so far published of the cool super-giant. It has no emission lines superimposed and a $\chi^{2}$ fitting to the same Munari et al. (2005) synthetic spectral library used in the automatic analysis of RAVE spectra suggests atmospheric parameters of $T_{\text {eff }}=3925 \pm 155,[\mathrm{M} / \mathrm{H}]=-0.39 \pm 0.26, \log g=2.87 \pm 0.91$, and a heliocentric radial velocity of $254.2 \pm 1.2 \mathrm{~km} \mathrm{~s}^{-1}$. The radial velocity reported by Schnurr et al. (2008) is $255 \mathrm{~km} \mathrm{~s}^{-1}$. The relatively large errors of the $\chi^{2}$ fitting are due to the contamination from the superimposed hot super-giant spectrum, whose main effect is to provide an overall veiling of the absorption lines. This has a particularly adverse effect on the wings of the CaII lines (most sensitive to $\log g$ ), amplified by the perturbation of the shape of the adjacent continuum by the wide underlying Paschen absorption lines of the hot component. The $\chi^{2}$ temperature would suggest a spectral type around K7, with the metallicity being appropriate for massive stars in the LMC.

\section{Conclusions}

The primary science driver for the RAVE survey is the investigation of the structure and evolution of the Milky Way. Nevertheless, as a natural byproduct of the survey, the resolution and diagnostic potential of the associated stellar spectra ensures a valuable resource for the stellar astrophysics community.

The present paper is the first in our series to explore the performance of RAVE in relation to the physics of peculiar stars, in particular, the Luminous Blue Variables of the Large Magellanic Cloud. The RAVE spectra provide a clear and comprehensive view of the LBVs, highlighting their temporal evolution and star-by-star differences. In particular, these have: $(i)$ extended the temporal baseline and wavelength domain over which the radial velocities of emission lines have been observed to remain constant, which reinforces the notion of an origin in a spherically symmetric component of the circumstellar environment, dynamically de-coupled from the central star; (ii) mapped the large variability of the absorption lines (both in radial velocity and profiles) in response to the "breathing" of the pseudo-photosphere forming in the heavy, out-flowing wind; (iii) documented the high variability and multi-shell nature of the wind P-Cyg absorption profiles; (iv) provided a clear recipe of the spectroscopic changes characterising the transition of LBVs from quiescence to outburst phases and vice-versa, in a close match to evidence gathered at more conventional (blue) optical wavelengths; $(v)$ offered the opportunity to test quantitatively the soundness of photoionisation modelling of the rich emission line spectrum observable over the RAVE range; (vii) discovered in R 127 the presence, and quantified the physical properties of, a massive detached ionised shell which was ejected during the 1982-2000 outburst.

Acknowledgements. The data were obtained as part of the RAVE survey using the UK Schmidt Telescope operated by the Anglo-Australian Observatory. Funding for RAVE has been provided by: the Anglo-Australian Observatory; the Astrophysical Institute Potsdam; the Australian National University; the Australian Research Council; the French National Research Agency; the German Research foundation; the Istituto Nazionale di Astrofisica at Padova; The Johns Hopkins University; the W. M. Keck foundation; the Macquarie University; the Netherlands Research School for Astronomy; the Natural Sciences and Engineering Research Council of Canada; the Slovenian Research Agency; the Swiss National Science Foundation; the Science \& Technoeached logy Facilities Council of the UK; Opticon; Strasbourg Observatory; and the Universities of Groningen, Heidelberg and Sydney. The RAVE web-site and associate database is accessible from www . rave-survey.org.

\section{References}

Allen, D. A., \& Glass, I. S. 1976, ApJ, 210, 666

Andrillat, Y., Jaschek, C., \& Jaschek, M. 1995, A\&AS, 112, 475

le Borgne, J.-F., Bruzual, G., Pelló, R., et al. 2003, A\&A, 402, 433

Breysacher, J., Azzopardi, M., \& Testor, J. 1999, A\&AS, 137, 117

Bowen, I. 1947, PASP, 59, 196

Castelli, F., \& Munari U. 2001, A\&A, 366, 1003

Castor, J. P., \& Lamers, H. J. G. L. M. 1979, ApJS, 39, 481

Cenarro, A. J., Cardiel, N., Gorgas, J., et al. 2001, MNRAS, 326, 959

Clark, J. S., Negueruela, I., Crowther, P. A., Goodwin, S. P., et al. 2005, A\&A, 434, 949

Cowley, A. P., \& Hutchings, J. B. 1978, PASP, 90, 636

Crowther, P. A. 2007, ARA\&A, 45, 177

Crowther, P. A., \& Willis, A. J. 1994, Sp. Sc. Rev., 66, 85

Crowther, P. A., Hillier, D. J., \& Smith, L. J. 1995, A\&A, 293, 172

Davidson K., Moffat, A. F. J., \& Lamers, H. J. G. L. M. 1989, ed. The Physics of Luminous Blue Variables, IAU Coll. 113 (Kluwer)

Davies, B., Oudmaijer, R. D., \& Vink, J. S. 2005, A\&A, 439, 1107

Feast, M. W., Thackeray, A. D., Wesselink, A. J. 1960, MNRAS, 121, 337

Ferland, G. J., Korista, K. T., Verner, D. A., et al. 1998, PASP, 110, 761

Garcia-Lario P., Riera A., \& Manchado A. 1998, A\&A, 334, 1007

Glass, I. S. 1984, MNRAS, 209, 759

Guo, J. H., \& Li, Y. 2007, ApJ, 659, 1563

Heydari-Malayeri, M., Meynadier, F., Walborn, N. R., et al. 2003, A\&A, 400, 923

Hubble, E., \& Sandage, A. 1953, ApJ, 118, 353

Humphreys, R. M., \& Davidson, K. 1994, PASP, 106, 1025

Jaschek, C., \& Jaschek, M. 1987, The Classification of Stars Stars, (Cambridge Univ. Press)

Jaschek, C., \& Jaschek, M. 1995, The Behaviour of Chemical Elements in Stars, (Cambridge Univ. Press)

Kenyon, S. J., \& Gallagher, J. S.-III 1985, ApJ, 290, 542

Lamers, H. J. G. L. M. 1995, in Astrophysical Application of Stellar Pulsation, ed. R. S. Stobie \& P. A. Whitelock, ASPC, 83, 176

Lamers, H. J. G. L. M., \& Cassinelli J. P. 1999, Introduction to Stellar Winds, Cambridge University Press

Lamers, H. J. G. L. M., \& Leitherer, C. 1993, ApJ, 412, 771

Lamers, H. J. G. L. M., Nota, A., Panagia, N., et al. 2001, ApJ, 551, 764

Langer, N., Garca-Segura, G., \& Mac Low, M.-M. 1999, ApJ, 520, L49

Leitherer, C., Damineli Neto, A., \& Schmutz, W. 1992, in Nonisotropic and Variable Outflows from Stars, L. Drissen, C. Leitherer and A. Nota eds., ASPC, 22, 1992

Lennon, D. J., Wobig, D., Kudritzki, R.-P., Stahl, O. 1993, Sp. Sci. Rev., 66, 207 Marrese, P. M., Boschi, F., \& Munari U. 2003, A\&A, 406, 995

Mateo, M. L. 1998, ARA\&A, 36, 435

Meynet, G., \& Maeder, A. 2005, A\&A, 429, 581

Munari, U. 1998, in Proccedings of the Gaia Workshop, V. Straizys ed., BalA, 8, 73

Munari, U. 2002, in Exotic Stars as Challenges to Evolution, IAU Coll. 187, ed. C. A. Tout \& W. Van Hamme, ASPC, 279, 25

Munari, U. 2003, in Gaia Spectroscopy, Science and Technology, ed. U. Munari, ASPC, 298, 227

Munari, U., \& Tomasella, L. 1999, A\&AS, 137, 521

Munari, U., Sordo, R., Castelli, F., \& Zwitter, T. 2005, A\&A, 442, 1127

Munari, U., Tomasella, L., Fiorucci, M., et al. 2008, A\&A, 488, 969

Nota, A., Pasquali, A., Drissen, L., et al. 1996, ApJS, 102, 383

Osterbrock, D. E., \& Ferland, G. J. 2006, Astrophysics of Gaseous Nebulae and Active Galactic Nuclei, 2nd edn., (Univ. Science Book)

Pauldrach, A. W. A., \& Puls, J. 1990, A\&A, 237, 409

Pojmanski, G. 2002, Acta Astron., 52, 397

Sanduleak, N., \& Philip, A. G. D. 1977, PASP, 89, 792

Schmutz, W., Leitherer, C., Hubeny, I., et al. 1991, ApJ, 372, 664

Schnurr, O., Moffat, A. F. J., St-Louis, N., Morrell, N. L., Guerrero, M. A., et al. 2008, MNRAS, 389, 806

Seabroke, G., Gilmore, G., Siebert, A., et al. 2008, MNRAS, 384, 11

Siebert, A., Bienaymé, O., Binney, J., et al. 2008, MNRAS, 391, 793

Siebert, A., et al. 2009, AJ, submitted

Sharov, A. S. 1975, in Variable stars and stellar evolution, (Reidel) IAU Symp, 67,275 
Smith, L. 1997, in Luminous Blue Variables: Massive Stars in Transition, ed. A Nota \& H. J. G. L. M. Lamers, ASPC, 120, 310

Smith, L. J., Nota, A., Pasquali, A., et al. 1998, ApJ, 503, 278

Smith, M. C., Ruchti, G. R., Helmi, A., et al. 2007, MNRAS, 379, 755

Smith, N., \& Owocki, S. P. 2006, ApJ, 645, L45

Smith, N., Vink, J. S., \& de Koter, A. 2004, ApJ, 615, 475

Stahl, O., \& Wolf, B. 1986, A\&A, 154, 243

Stahl, O., Wolf, B., Klare, G., et al. 1983, A\&A, 127, 49

Stahl, O., Wolf, B., Leitherer, C., et al. 1984, A\&A, 140, 459

Stahl, O., Wolf, B., Leitherer, C., et al. 1985, A\&AS, 61, 237

Stahl, O., Jankovics, I., Kovács, J., et al. 2001, A\&A, 375, 54

Steinmetz, M., Zwitter, T., Siebert, A., et al. 2006, AJ, 132, 1645

Stothers, R. B., \& Chin, C.-W. 1995, ApJ, 451, L61

Strittmatter, P. A., Woolf, N. J., Thompson, R. I., et al. 1977, ApJ, 216, 23

Szeifert, Th., Humphreys, R. M., Davidson, K., et al. 1996, A\&A, 314, 131

Thackeray, A. D. 1965, MNRAS, 129, 169
Thackeray, A. D. 1974, MNRAS, 168, 221

Trundle, C., Kotak, R., Vink, J. S., et al. 2008, A\&A, 483, L47

van Genderen, A. M. 2001, A\&A, 366, 508

Veltz, L., Bienaymé, O., Freeman, K. C., et al. 2008, A\&A, 480, 753

Venn, K. A. 1997, in Luminous Blue Variables: Massive Stars in Transition, ed.

A. Nota \& H. J. G. L. M. Lamers, ASPC, 120, 95

Vink, S. J. 2008, NewAR, 52, 419

Vink, J. S., \& de Koter, A. 2002, A\&A, 393, 543

Vink, J. S., de Koter, \& A., Lamers, H. J. G. L. M. 1999, A\&A, 350, 181

Walborn, N. R. 1977, ApJ, 215, 53

Walborn, N. R., Stahl, O., Gamen, R. C., et al. 2008, ApJ, 683, L33

Weis, K. 2003, A\&A, 408, 205

Wesselink, A. J. 1956, MNRAS, 116, 3

Wolf, B. 1989, A\&A, 217, 87

Zwitter, T., Siebert, A., Munari, U., et al. 2008, AJ, 136, 421 